\title{
Recommendation to wear gloves wet with diluted detergent for preventing COVID-19 transmission
}

\author{
Daisuke Miyazawa ${ }^{1}$ \\ ${ }^{1}$ Affiliation not available
}

August 25, 2020

Contact transmission of severe acute respiratory syndrome coronavirus 2 (SARS-CoV-2) can occur when contaminated hands touch the mucosa of the mouth, nose, or eyes. Contaminated hands also facilitate indirect contact transmission of the virus from one fomite surface to another. Consequently, hand hygiene is important to prevent the spread of SARS-CoV-2. However, changing plastic gloves or sanitizing hands every time one touches possibly contaminated fomites will need to be frequently performed and is practically impossible. The Japanese Ministry of Economy, Trade and Industry recently reported that SARS-CoV-2 was deactivated by several diluted surfactants ${ }^{1}$. Wearing cloth gloves wet with a diluted detergent will prevent infection because the virus gets deactivated within a short period of time on these gloves. The virus gets deactivated within a short period of time on these gloves. Unlike ethanol, detergents are not volatile. Thus, gloves can remain wet and effective for long periods of time. Although the thin diluted detergent could remain on the touched sites, it can be rinsed under running water or wiped afterwards, if necessary. In case of contamination, the detergent remaining on the fomites may also deactivate the virus. To avoid skin contact with the detergent, a plastic glove can be worn underneath the wet cloth glove. This method may be especially effective for healthcare workers who deal with COVID-19 patients or people in the city who handle fomites that many people touch. Additionally, wearing gloves wet with diluted detergent may prevent the user from touching his face, which would further prevent contact transmission.

\section{Reference}

1. Ministry of Economy, Trade and Industry, Surfactants and Hypochlorous Acid Solution for Removal of Coronavirus from Surfaces (Final Announcement), 26 June 2020, www.meti.go.jp/english/press/2020/0626_004.html. 